\title{
Aprendizagem organizacional e práticas ambientalmente sustentáveis em instituições de ensino superior: um estudo sobre o Canadá e o Brasil
}

\section{Sandra Simm Rohrich ${ }^{1}$ e Adriana Roseli Wünsch Takahashi $^{2}$}

${ }^{1}$ Universidade Federal do Paraná. Setor Litoral. Rua Jaguariaíva, 512. Caiobá. Matinhos-PR, Brasil (CEP 83260-000). E-mail: sandrasimm@ufpr.br.

${ }^{2}$ Universidade Federal do Paraná. Departamento de Administração. Av. Pref. Lothario Meissner, 3400. Jardim Botânico. Curitiba-PR, Brasil, (CEP 80210-170).

Resumo. Este artigo teve por objetivo descrever a sustentabilidade ambiental em instituições de ensino superior canadenses e brasileiras a partir da aprendizagem baseada em práticas, oriunda da intersecção entre a aprendizagem formal e informal. A metodologia que norteou o estudo foi de natureza descritiva qualitativa, relatando experiências de instituições de ensino superior com a sustentabilidade, no Canadá e no Brasil. 0 estudo foi permeado pelo modelo teórico dos processos formais e informais de aprendizagem interligados. A sustentabilidade nas IES tem sido um tema de importância crescente. Assim como nos demais setores organizacionais, as instituições de ensino superior também são responsáveis pela sustentabilidade em suas perspectivas econômica, social e ambiental. Como principais resultados foi destacada a importância dos modelos de indicadores formais para padronizar as ações de sustentabilidade nas instituições de ensino superior. Contudo, são as práticas das instituições de ensino superior que traduzem as suas ações reais para a sustentabilidade, resultantes da intersecção entre a aprendizagem formal e a aprendizagem informal. Foi possível perceber que as instituições de ensino superior que adotam um mesmo modelo de indicadores teriam pontos comuns no processo de aprendizagem, pois a aprendizagem formal, obtida por treinamentos e padronização, tornaria as práticas mais homogêneas. A partir destes estudos sobre a sustentabilidade ambiental em instituições de ensino superior no Canadá e no Brasil, considera-se que estas instituições estão evoluindo em relação à adoção de práticas sustentáveis.

Palavras-chave: Sustentabilidade; Instituição de Ensino Superior; Práticas.

Abstract. Organizational learning and environmentally sustainable practices in college education institutions: $A$ study

ISSN 2359-1412/RBGAS-2020-0039/2020/7/15/28/387

Rev. Bras. Gest. Amb. Sustent.

http://revista.ecogestaobrasil.net 
about Canada and Brazil. This article aimed to describe the environmental sustainability in Canadian and Brazilian higher education institutions starting from learning based on practices, derived from the intersection between formal and informal learning. The methodology which guided the study was made of by qualitative descriptive nature, reporting experiences of higher education institutions with sustainability, in Canada and Brazil. The study was permeated by the theoretical model of the formal and informal processes of learning linked. Sustainability on the higher education institutions has been being a growing importance theme. As well as in other organizational sectors, the higher education institutions are also responsible for the sustainability in its economical, social and environmental perspectives. As main results was the importance of formal indicators to standardize the actions of sustainability at the higher education institutions was highlighted. Yet, the higher education institutions practices translate its real actions to sustainability, resulting from the intersection between formal learning and informal learning. It was possible to notice that the higher education institutions which adopt the same model of indicators would have common points in the learning process, because formal learning, obtained by trainings and standardization, would make practices more homogeneous. Starting from these studies about environmental sustainability in higher education institutions in Canada and in Brazil, it is considered the higher education institutions are evolving about the adoption of sustainable practices.

Keywords: Sustainability; College education; Practices.

\section{Introdução}

A sustentabilidade nas Instituições de Ensino Superior (IES) tem sido um tema de importância crescente, assim como nos demais setores organizacionais. Desde a década de 1970, encontros internacionais têm motivado os países desenvolvidos e em desenvolvimento a minimizarem os seus impactos ambientais e consumirem menos recursos naturais. Em trajetória semelhante, as IES também são responsáveis pela sustentabilidade no desenvolvimento, por isso, desde 1990, a partir da Declaração de Talloires, precisaram começar a aprender a lidar com este tema. Outros compromissos internacionais vêm firmando o interesse das IES com a sustentabilidade, assim foi com a Declaração de Halifax em 1991. Em 1993, a Declaração de Swansea foi inspirada nos exemplos de Talloires e Halifax. Em 1994, foi escrita a Carta Copérnicus, uma carta universitária para o Desenvolvimento Sustentável, elaborada pela Associação das Universidades Européias. Em 1995 foi constituída, em São José na Costa Rica, a Organização Internacional de Universidades pelo Desenvolvimento Sustentável e Meio Ambiente (OIUDSMA, 2002). Em 2000 foi criada a Global Higher Education for Sustainability Partnership (GUESP). Em 2005, a ONU criou a Década da Educação para o Desenvolvimento Sustentável. Em 2007, foi desenvolvida a iniciativa Principles for Responsible Management Education (PRME) sob coordenação do Pacto Global das Nações Unidas (Termignoni, 2012). Ainda nesta perspectiva, desde 2006 existe na Europa o projeto Ecocampus (Tauchen e Brandli, 2006). Também em 2006 foi oficialmente lançada 
a Association for the Advancement of Sustainability in Higher Education (AASHE), a primeira associação profissional norte-americana com interesse no avanço da sustentabilidade no campus (AASHE, 2015). Sendo o Canadá um dos países membros da AASHE, é nesta direção que esse estudo se fundamenta, analisando o processo de aprendizagem para a sustentabilidade nas IES canadenses e também no Brasil, descrevendo assim as realidades de cada país. Considera-se, para tanto, a aprendizagem formal como aquela que ocorre para atender as políticas públicas ou indicadores com estrutura pré-definida, por meio de mecanismos formais como treinamento, cursos, entre outros. A aprendizagem informal está calcada no conhecimento prático, em soluções encontradas dentro das IES para resolver os seus problemas relativos à sustentabilidade, portanto, não demanda treinamento formal. Assim, este artigo tem como objetivo: Descrever a sustentabilidade ambiental em IES canadenses e brasileiras a partir da aprendizagem baseada em práticas, oriunda da intersecção entre a aprendizagem formal e informal (Flach e Antonello, 2011). A escolha pelo Canadá reside na condição deste país, assim, como o Brasil, apresentar práticas sustentáveis diversificadas, não havendo uma orientação única para a sustentabilidade em termos de indicadores e diretrizes escolhidos pelas IES (Fonseca et al., 2011).

Entenda-se aqui que a sustentabilidade está enfatizada na sua dimensão ambiental, não vindo para o debate outras dimensões colocadas por Sachs (1993), como a social, econômica, ecológica, espacial e cultural, e por Elkington (1999), como a econômica, social e ambiental, denominado por este de Triple Botton Line.

\section{Aprendizagem organizacional}

A literatura sobre aprendizagem organizacional tem avançado desde os primeiros estudos nos anos 1950 (Easterby-Smith e Araújo, 2001), sendo Cyert e March (1963) os primeiros autores a referenciar a aprendizagem organizacional, ainda dentro de um enfoque adaptativo (Cyert e March, 1963; Lyles e Easterby-Smith, 2003). Outros autores fizeram contribuições relevantes para o campo como os constructos de aprendizagem organizacional baseados no processamento de informações (Huber, 1991), os conceitos de exploration e exploitation e a importância do equilíbrio para inovar (March, 1991), a interação entre a aprendizagem organizacional e a Psicologia (Cohen, 1991), a aprendizagem e seus ciclos (Argyris e Schon, 1978), dentre outros clássicos, de fundamentação e populares (Easterby-Smith e Lyles, 2003).

Dentre as perspectivas diversas de pesquisa com que os autores vêm tratando o tema, destacam-se a comportamental, a cognitiva e a cultural (Tsang, 1997). Esta diversidade de definições e perspectivas foi observada por Nicolini e Meznar (1995), que sugeriram uma conceitualização que integrasse as várias perspectivas. Os autores consideraram a aprendizagem organizacional como uma construção social que transforma a cognição adquirida em conhecimento abstrato responsável; assim, o quadro referencial para investigar a aprendizagem organizacional deveria ser expandido para promover um melhor entendimento do fenômeno, pois interpretações restritivas ou suposições afetam o caminho no qual a aprendizagem foi concebida, frequentemente limitando a perspectiva sobre o fenômeno. Assim, uma perspectiva integrada, no entanto, pode ser mais rica ao entender que estas dimensões não se encontram separadas, e que todas estão envolvidas em um processo de aprendizagem (Takahashi, 2007).

Esta perspectiva integrada é aqui assumida, pois entende-se que a aprendizagem organizacional envolve um processo integrado e cultural (Cook e Yanow, 1993), que se refere a aquisição, sustentação ou compartilhamento de significados intersubjetivos, o que ocorre na interação social por meio de mecanismos formais e informais. Portanto, inclui valores, mudanças no comportamento ou potencial de comportamento, e mapas mentais. Isso porque o conhecimento organizacional pressiona a ligação entre ação, contexto e processos, e envolve um fluxo de criação, utilização e institucionalização (Patriotta, 2003). 
Hedberg (1981) aponta que organizações não possuem cérebros, mas têm sistemas cognitivos e memórias, e que desenvolvem também uma visão de mundo e ideologias. Essa memória organizacional mantém certos comportamentos, mapas mentais, normas e valores todo o tempo, apesar da entrada e saída de funcionários e de líderes. Assim, cultura, história, conhecimento e mudanças importam no processo de aprendizagem (Elkjaer, 2003). Barr et al. (1992) argumentam que a renovação estratégica requer ajustes nos modelos mentais acompanhando as mudanças no ambiente, pois os modelos mentais dos gestores facilitam e limitam a atenção para codificar e salientar a informação sobre mudanças em ambientes organizacionais.

Quanto aos níveis de aprendizagem, a literatura tem mostrado que ela pode ocorrer em nível individual, grupal, organizacional ou interorganizacional. Porém, o conceito de aprendizagem em uma ótica dinâmica que incorpora a noção de mudança contínua, integra sistemicamente estas esferas (Antonello, 2005). A questão, portanto, não está em definir o ponto de inflexão entre níveis, mas sim analisar se houve aprendizagem, de que forma ocorreu e quando é organizacional (Takahashi, 2007).

Em suma, este trabalho situa-se no campo da teoria social de aprendizagem, que considera a participação no processo social, envolvendo o ser e o tornar-se. Neste contexto, uma situação coloca certas possibilidades para determinadas ações e restringe outras, o que depende das experiências, da vivência, da interação social, da distribuição do poder. Desta forma, os autores de aprendizagem selecionados são os que convergem com esta concepção.

\section{Aprendizagem informal.}

A aprendizagem formal é estruturada, institucional, realizada em sala de aula e com o auxílio de um instrutor. Já a aprendizagem informal abrange oportunidades naturais do cotidiano, de tal modo que o processo de aprendizagem é controlado pelo indivíduo. Caracteriza-se ainda como experiencial, prática e não-institucional (Conlon, 2004; Flach e Antonello, 2011). Para Flach e Antonello (2011) a aprendizagem informal contrasta com as atividades em sala de aula, que envolvem conhecimentos abstratos. No ambiente de trabalho a aprendizagem informal resulta da integração de funcionários, tarefas diárias, oportunidades, eventos, rotinas, interações com a cultura e o ambiente externo, situações que fogem do planejamento e procedimentos do cotidiano (Flach e Antonello, 2011).

A aprendizagem informal é baseada na experiência intencional, porém sem ter sido formalmente estruturada: induzida por um processo de reflexão crítica, ação, próatividade e criatividade. Pode ser planejada ou não, embora envolva algum grau de consciência de quem aprende (Watkins e Marsick, 1992; Marsicke e Watkins, 2001; Reatto e Godoy, 2015).

Para Antonello e Godoy (2009), o debate acerca da natureza da aprendizagem nas organizações vem crescendo e novos temas têm surgido, como a aprendizagem estratégica e internacional e os estudos envolvendo as práticas. Para as autoras, a corrente de pensamento da aprendizagem baseada em práticas parece contemplar, mais proximamente, as questões levantadas. Portanto, o desenvolvimento de estudos com a adoção de uma perspectiva baseada em práticas permite um olhar para os microprocessos que permeiam as práticas contínuas dos atores de um sistema social. Este é o tema da próxima sessão.

\section{Aprendizagem baseada em práticas.}

Na linguagem cotidiana o termo "prática" apresenta diferentes significados. Pode expressar alguma coisa concreta ou real, frequentemente em oposição a alguma coisa abstrata ou teórica. Assim, a perspectiva de práticas está inserida principalmente dentro de uma aproximação sociológica de aprendizagem organizacional que considera 0 
conhecimento como alguma coisa que as pessoas fazem juntas, onde o saber e o fazer estão enredados (Gherardi, 2011).

A lente das práticas pode auxiliar na explicação de fenômenos sociais em um caminho processual, sem perder o contato com a natureza mundana da vida cotidiana e a natureza concreta e material das atividades (Nicolini, 2013). Antonello e Godoy (2010) sugerem a abordagem da aprendizagem baseada em práticas como uma possibilidade integradora. Para Flach e Antonello (2011) um elemento importante para a análise de processos informais de aprendizagem consiste no conceito de aprendizagem situada, no qual as pessoas aprendem por meio de observação e interação com os membros do grupo social (Lave e Wenger, 1991). A interação social é um componente crítico da aprendizagem situada e o aprendizado ocorre de maneira não intencional, não deliberada, um processo mediador importante é o da participação em grupos denominados Comunidades de Prática - COPs (Elkjaer, 2011).

Gherardi (2012) esclarece que o conceito de "comunidade de práticas" surgiu pela primeira vez dentro de análises da aprendizagem organizacional como o precursor para estudos sobre o conhecimento prático e como ele é criado, disseminado e conservado em práticas de trabalho e na comunidade que se forma em torno de uma prática. A autora faz referência a um quebra-cabeças ao argumentar que todos aqueles que interagem dentro de uma específica prática de trabalho possuem diferentes "peças" do conhecimento que deve ser montado em conjunto para adquirir inteligibilidade.

As práticas aqui consideradas no estudo do processo de aprendizagem são as práticas de sustentabilidade, mais especificamente em IES. Neste recorte, as mudanças e o conhecimento organizacional são os pilares para um processo de aprendizagem em nível organizacional.

\section{Procedimentos metodológicos}

A metodologia que norteou o estudo exposto é de natureza descritiva qualitativa. Pesquisas qualitativas são as que têm por objetivo estudar situações complexas ou estritamente particulares (Richardson, 1999). Operacionalmente trata-se de uma pesquisa fundamentada em dados secundários, relatando experiências de IES com a sustentabilidade, no Canadá e no Brasil. A escolha pelo Canadá deu-se devido às práticas sustentáveis naquele país serem incomuns e muito diversificadas, não havendo uma orientação única para a sustentabilidade em termos de indicadores e diretrizes escolhidos pelas IES (Fonseca, 2011). Em face da realidade apresentada esse artigo tem por objetivo Descrever a sustentabilidade ambiental em IES canadenses e brasileiras a partir da aprendizagem baseada em práticas, oriunda da intersecção entre a aprendizagem formal e informal. 0 estudo tem como questão de pesquisa relatar as práticas de sustentabilidade presentes nas IES localizadas no Canadá e no Brasil, evidenciando a aprendizagem formal e as práticas de ambos os países.

Para o contexto canadense foi empregado como fonte para a pesquisa o International Journal of Sustainability in Higher Education. A busca foi realizada nas edições do periódico a partir do ano 2000. Foram relacionados os artigos nos quais há menção a alguma instituição de ensino superior Canadense no título e também à sustentabilidade ou gestão ambiental, ou relatórios de sustentabilidade ou relatório ambiental. No resumo dos artigos foi possível identificar a perspectiva de práticas sustentáveis nos campi. Cumprindo esses requisitos foram selecionados seis artigos.

Para ilustrar o contexto nacional a busca pelos artigos foi realizada no site de periódicos da CAPES. Foram mantidos seis artigos considerados mais pertinentes ao estudo, igualando o número de contribuições oriundas do Canadá e do Brasil. Do mesmo modo que na seleção das IES canadenses, buscou-se artigos que fizessem menção a alguma 
IES no título e também à sustentabilidade, gestão ambiental, relatórios de sustentabilidade ou relatório ambiental.

Além dos artigos selecionados para o Canadá e para o Brasil, o estudo foi permeado pelo modelo teórico de Flach e Antonello (2011), no qual os autores citam que os processos formais e informais de aprendizagem estão sempre interligados, sendo que ambos possuem importante papel no desenvolvimento cognitivo e das capacidades dos indivíduos. Os autores mencionaram Svensson et al. (2004) e como as competências podem ser desenvolvidas quando existe um arranjo eficiente entre aprendizagem formal e informal, proporcionando espaço para a criação a partir de conhecimentos práticos e teóricos. Assim, a partir da proposição desses autores e da noção de aprendizagem baseada em práticas (que é predominantemente informal), pode-se dizer que ocorre o desenvolvimento de capacidades dos indivíduos quando se sobrepõe e/ou interconectam processos formais e informais de aprendizagem (Figura 1).

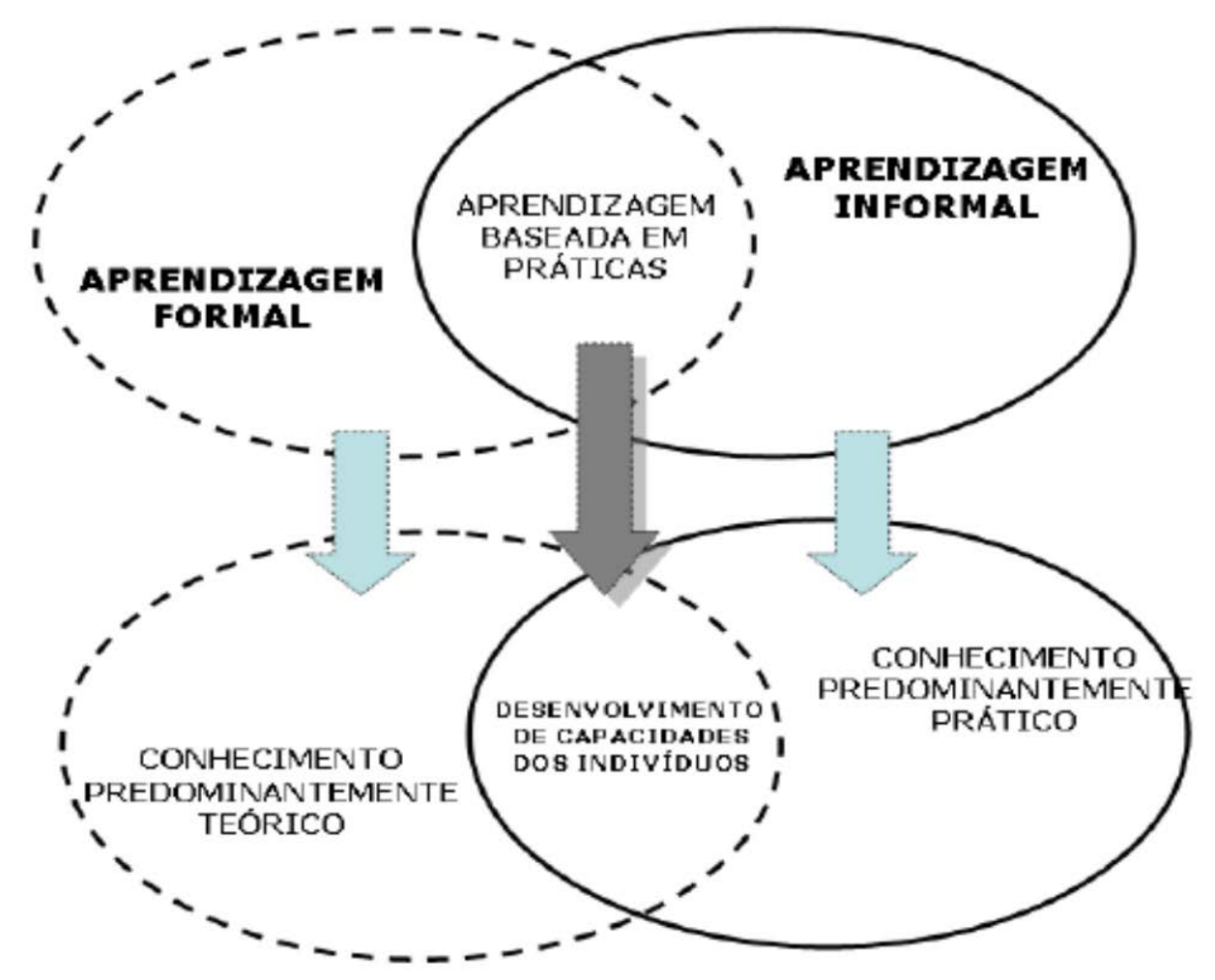

Figura 1. Interação entre processos formais e informais de aprendizagem. Fonte: Elaborado por Flach e Antonello (2011), a partir de Svensson et al. (2004), Conlon (2004) e Nicolini et al. (2003).

\section{Resultados}

Práticas sustentáveis nas IES canadenses

No Canadá, o atendimento a indicadores pré-definidos e outras ações que dependem de formalizações mediante documentos e treinamento de colaboradores, compõem a aprendizagem formal. As práticas surgem mediante uma composição do aprendizado formal e do informal, como resultado da experiência coletiva que promovem 
o desenvolvimento de capacidades específicas. Originárias da aprendizagem situada, das relações sociais voltadas à sustentabilidade, que se repetem e se legitimam entre os pares.

Nos próximos parágrafos são relatadas, em ordem cronológica, experiências de IES canadenses para avaliar as suas práticas sustentáveis. Esses estudos relatam a aplicação de indicadores formais como: o Campus Sustainability Assessment Framework (CSAF) relatado por Beringer (2006); Clarke (2006) que fez uma relação entre o conceito de estratégia deliberada e emergente (Mintzberg) e Interação entre planejamento e implementação; (Andrews) que abordou sobre as práticas sustentáveis; Mitchell (2011) que também seguiu uma proposta de práticas sustentáveis, sem uma estrutura formal a ser seguida; a formalização foi relatado por Kamal e Asmuss (2013) que avaliaram, em estudo de benchmarking, qual seria o modelo de indicadores mais adequado para a sustentabilidade em IES, para isso fizeram um estudo em quatro modelos; Bilodeau, Podger, e Abd-El-Azi (2014), assim como Mitchell (2011) avaliaram a sustentabilidade sem usar um modelo específico; e por fim, Munro, Marcus, Dolling, Robinson e Wahl (2016) escreveu a parceria para a sustentabilidade entre a cidade de Vancouver e a Universidade da Columbia Britanica. A seguir segue detalhamento dos estudos citados.

Beringer (2006) introduziu a Campus Sustainability Assessment Framework (CSAF) como uma metodologia de auditoria sustentável em um campus. 0 autor descreveu o projeto Sierra Youth Coalition Sustainable Campus, como uma campanha que acontece no Canadá para a sustentabilidade no Campus, empregando o modelo CSAF, que já havia sido implementado na University of Prince Edward Island (UPEI) para facilitar um projeto de educação para a sustentabilidade. 0 estudo acompanha a experiência utilizando a CSAF para conduzir a primeira auditoria de sustentabilidade no campus.

Clarke (2006) se propôs a desafiar a abordagem de estratégia deliberada para o SGA e ofereceu um modelo baseado tanto na realidade prática experienciada pela Universidade de Dalhousie, Canadá, quanto na teoria da estratégia emergente. Para tal, o estudo se fundamentou em Mintzberg $(1978,1994)$ e Andrews (1987). A autora observou que para os Sistemas de Gestão Ambiental o modelo de estratégias emergentes é mais adequado para a realidade do que a abordagem de estratégia deliberada, compondo a aprendizagem informal. Concluíram que teoricamente o SGA inclui fases que seguem uma a outra sequencialmente, mas quando consideram a teoria da estratégia emergente (Mintzberg) e a noção de inter-relação entre as fases de planejamento e implementação (Andrews) entendem que os modelos de SGA deveriam incluir estratégias emergentes e não realizadas como parte do ciclo e que a separação entre planejamento e implementação é inexistente. Dois exemplos de planos emergentes observados foram as políticas de compras sustentáveis e as iniciativas de compostagem.

Mitchel (2011) relatou a preparação do campus principal da universidade do Brock, St. Catherine, Ontário, Canadá, para uma auditoria de sustentabilidade. 0 estudo ofereceu evidências qualitativamente analisadas de que campi pequenos e médios no Canadá, com orçamentos limitados podem ter sucesso em mover-se para iniciativas sustentáveis. Sem uma estrutura formal de governança ou um quadro teórico de referência, um amplo espectro de definições de sustentabilidade foram citadas pelos stakeholders, abrangendo o enfoque ambiental, social ou econômico, prevalecendo a combinação entre as três. Foram observadas iniciativas incluindo a co-geração de energia, as construções verdes de novos edifícios e a reciclagem. De acordo com Mitchel (2011) a promoção da consciência sobre o meio ambiente engloba questões de justiça social e a demonstração de que boas práticas podem ser promovidas pelas IES.

Kamal e Asmuss (2013) realizaram um estudo na Universidade de Saskatchevan, onde conduziram um benchmarking para modelos de indicadores de sustentabilidade. Avaliaram quatro modelos utilizados na América do Norte e Canadá: 0 primeiro foi o Sustainability Assessment Questionarie (SAQ), que consiste em um questionário elaborado pelo University Learder for a Sustainable Future, nos EUA. Tem como propósito mensurar o 
envolvimento das IES com a sustentabilidade. Avalia 7 dimensões: currículo, pesquisa e bolsa de estudos; operação; desenvolvimento do corpo docente; serviço; oportunidades para estudantes; e administração, missão e planejamento. 0 segundo modelo avaliado foi o Campus Sustainability Assessment Framework (CSAF). A ferramenta contém 170 indicadores organizados em 10 dimensões. Abrange, portanto, as dimensões SOCIAIS (conhecimento, comunidade, governança, economia e riqueza, e saúde e bem-estar) e as dimensões AMBIENTAIS (ar, água, terra, materiais e energia). 0 terceiro foi o College of Sustainability Report Card (CSRC) é uma iniciativa do Sustainaible Endowments Institute, uma organização sem fins lucrativos envolvida com pesquisa e educação para avançar na sustentabilidade nas operações dos campi e nas práticas de doações. Foi desenhado para identificar IES líderes em seus compromissos com a sustentabilidade. São 43 indicadores em nove dimensões: administração; mudanças climáticas e energia; alimentação e reciclagem; construções verdes; envolvimento dos estudantes; transportes; transparência nas doações; prioridades de investimentos; e envolvimento dos acionistas. O quarto modelo avaliado foi o Sustainability Tracking Assessment and Rating System (STARS), que abrange cinco dimensões acadêmico envolvimento, operações, planejamento e inovação, bem como 19 indicadores. O STARS compõe o programa da Association for Advancement of Sustainability em Higher Education (AASHE), oferecendo apoio aos campi dos EUA, México e Canadá, provendo recursos e suporte para os esforços em torno da sustentabilidade. 0 STARS foi identificado pelos autores como o modelo mais efetivo para analisar a sustentabilidade em todas as áreas do Campus na Universidade de Saskatchevan, esta possui atualmente dois compromissos com a sustentabilidade: o acadêmico e a administração.

Em uma perspectiva informal da aprendizagem, sem adotar um modelo específico de indicadores, Bilodeau et al. (2014), buscaram descrever os avanços no campus da Universidade de British Columbia Okanagan, no Canadá, considerando os objetivos operacionais e acadêmicos. Indicaram que a sustentabilidade pode ser vista como uma direção para a inovação, resultando em operações mais eficientes e processos de negócios, além de reduzir os custos e impactos ambientais. Segundo os autores o campus tem estabelecido parcerias para avançar em pesquisas inovadoras e soluções sustentáveis para uma agenda ambiental. Essas parcerias têm sido dirigidas pelos compromissos da liderança com a sustentabilidade; questões econômicas; requerimentos regulatórios e legislativos; e regras da sociedade.

Munro et al. (2016), também sem utilizarem indicador específico, descreveram o programa de estágio curricular do Greenest City Scholars. Neste programa os estudantes da Universidade da Columbia Britanica trabalham em projetos na cidade de Vancouver e ajudam a avançar nas metas de sustentabilidade. Os autores relataram os sucessos, desafios e lições aprendidas no programa. A parceria com a Cidade de Vancouver foi formalizada em um memorando de entendimento. O programa teve início em 2010, o memorando estabeleceu um conjunto de princípios para apoiar os interesses compartilhados relativos aos objetivos e ações para a sustentabilidade na Universidade e os esforços da cidade de Vancouver em um Plano de Ação para uma cidade ecológica. A duração do Memorando tem a previsão de 20 anos (2010-2020).

\section{Práticas sustentáveis nas IES brasileiras}

No Brasil, como aspecto comum dentre as realidades observadas, a ISO 14001 apareceu como norteadora para as IES sendo a diretrizprecursora para a aprendizagem formal. Além da ISO 14001 a Agenda Ambiental da Administração Pública (A3P), proposta pelo Ministério do Meio Ambiente (MMA), tem sido uma importante referência, principalmente para as Instituições públicas. Além disso, em 2017 a A3P desenvolveu um indicador específico para as IES, denominado Gestão Socioambiental nas IES públicas. 
Neste contexto o panorama nacional trouxe diferentes perspectivas, conforme indicadas a seguir: Engelman et al. (2009) realizaram um estudo de casos múltiplos para descrever as práticas em quatro instituições no Rio Grande do Sul, todas fundamentadas na ISO 14001 (ABNT, 2015) para nortear as suas práticas; Kruger et al. (2011) fizeram uso da A3P, o estudo foi em uma universidade do município de Chapecó, estado de Santa Catarina; dando continuidade ao trabalho de Engelman et al. (2009), Machado et al. (2013) aplicaram uma survey em setenta e cinco IES. A ISO 14001 (ABNT, 2015) foi empregada para elaborar uma lista de práticas que foram verificadas nas IES; Sgarbi et al. (2013) descreveram o caso da Universidade Federal do Rio Grande do Sul, onde foi desenvolvido um modelo de indicadores denominado Levantamento de Aspectos e Impactos Ambientais (LAIA). Rocha et al. (2015) analisaram as práticas de uma IES no Estado de Santa Catarina, utilizando um modelo denominado Sistema Contábil Gerencial (SICOGEA). Peixoto et al. (2019) investigaram as práticas sustentáveis adotadas pelos técnicos administrativos da Universidade Federal Rural de Pernambuco, Unidade Acadêmica de Garanhuns (UFRPE/UAG), a partir dos eixos propostos pela A3P. A seguir melhor detalhamento desses trabalhos.

Engelman et al. (2009) desenvolveram estudo no qual descreveram as práticas sustentáveis em quatro IES no estado do Rio Grande do Sul. Os resultados mostraram que, um grande número de práticas é empregada e que apesar de nem todas estarem em busca de certificações, todas se baseiam na norma ISO 14001 (ABNT, 2015). Identificaram que todas as IES desenvolvem as seguintes práticas: diagnóstico dos impactos significativos para o meio ambiente; disseminação dos projetos ambientais desenvolvidos dentro da instituição; controle de efluentes; controle de resíduos; programa de seleção de lixo e espaços verdes. As práticas formais observadas nos campi abrangem a constituição de departamentos ou áreas para a gestão; cursos de formação de gestores ambientais, bem como treinamento e sensibilização da equipe de funcionários; inclusão nos currículos de conteúdos sobre sustentabilidade; desenvolvimento de projetos de pesquisa sobre a sustentabilidade do meio ambiente; organização de eventos sobre a questão ambiental.

Kruger et al. (2011) consideraram buscaram analisar a aderência de uma instituição comunitária de ensino superior à A3P. Para atingir o objetivo proposto a pesquisa comparou as demandas da $\mathrm{A} 3 \mathrm{P}$ às práticas de gestão socioambiental desenvolvidas pela IES. A IES está situada na região oeste do Estado de Santa Catarina, no Município de Chapecó. Foram pontuadas as ações e práticas da IES de acordo com o atendimento às exigências da $\mathrm{A} 3 \mathrm{P}$, que apresenta ainda cinco eixos temáticos, atendidos em grande parte pelas práticas. Para o primeiro eixo, uso racional dos recursos naturais e bens públicos, a IES faz acompanhamento semestral para reduzir o consumo de papel e de energia. Realiza ainda a captação da água da chuva, um projeto de substituição do uso de copos plásticos e uma central de resíduos. Para o segundo eixo, gestão adequada dos resíduos gerados, há um programa de reciclagem e destinação adequada dos resíduos com uma central para cada tipo de resíduo e também uma central de reagentes e resíduos químicos. Para o eixo 3, qualidade de vida no ambiente de trabalho, foi observado um programa permanente de ginástica laboral, acompanhamento da CIPA, ponto eletrônico, banco de horas e programa de conscientização contra o alcoolismo, programa de saúde ocupacional e um programa permanente de políticas de recursos humanos. No eixo 4, sensibilização e capacitação dos servidores, há os programas Metamorfose e Marco de uma Trajetória, destinados aos discentes. Com a comunidade do entorno a IES desenvolve um programa de orientação profissional, projetos e programas de extensão universitária e de assistência social. Para o eixo 5, licitações sustentáveis, a IES não descreveu nenhuma prática.

Dando continuidade ao trabalho de Engelman et al. (2009) e Machado et al. (2013) tiveram por objetivo identificar práticas de gestão ambiental realizadas nas universidades brasileiras tendo como base uma survey em 75 universidades. 0 estudo evidenciou que, 
em um total de 30 práticas que compuseram a survey, 13 práticas são realizadas por grande parte das instituições no país, alcançado alta adoção de acordo com as respostas obtidas na pesquisa. Dez práticas alcançaram média adoção por parte das IES. Finalmente, em sete práticas as universidades apresentaram baixa adesão. Outro resultado relevante foi que o grupo de universidades que manifestou maior média em grande parte das práticas foram as universidades que possuem departamentos específicos para a gestão ambiental (40\%).

Sgarbi et al. (2013) descreveram o caso da Universidade Federal do Rio Grande do Sul (UFRGS), na qual foi desenvolvida um modelo denominado LAIA (Levantamento de Aspectos e Impactos Ambientais) para auxiliar na implantação do Sistema de Gestão Ambiental (SGA). O modelo carrega conceitos da ISO 14001 e adaptações da Failur mode and effect analysis (FMEA). Foram elaborados diagnósticos para os prédios da Universidade em relação aos seus potenciais impactos ambientais. Além do LAIA, no SGA da UFRGS existem mais três programas transversais que atingem toda a universidade, são eles: Educação ambiental; Licenciamento ambiental;e e Certificação ambiental. Articulados com esses quatro programas há ainda na UFRGS dezesseis projetos temáticos que orientam a implantação do SGA. São realizados em conjunto com diversos setores que buscam alternativas mediante práticas como: redução do consumo de água e energia elétrica; disposição e tratamento adequado de resíduos; realização de licenças e laudos ambientais, entre outros.

Rocha et al. (2015) tiveram por objetivo analisar a sustentabilidade ambiental de uma IES do estado de Santa Catarina. A IES não possui Sistema de Gestão Ambiental, tampouco qualquer outro tipo de treinamento ou ação formal para a sustentabilidade, se restringindo à gestão de resíduos, conforme determina a legislação. 0 estudo foi desenvolvido por meio de questionário enviado aos responsáveis por três setores da IES, com o propósito de responder a uma lista de verificação com 154 questões propostas e agrupadas em nove dimensões, fornecedores/compras, ecoeficiência do processo de prestação de serviços, prestação de serviço-atendimento aos acadêmicos, responsabilidade social, gestão estratégica, indicadores gerenciais, recursos humanos, indicadores contábeis e auditoria ambiental. Esse questionário seguiu um modelo denominado de Sistema Contábil Gerencial (SICOGEA).

Peixoto et al. (2019) notaram que na UFRPE/UAG já existem várias iniciativas pontuais e individuais de ações sustentáveis no ambiente de trabalho. Porém, não perceberam nenhuma política institucional que vise o desenvolvimento sustentável dentro da organização, apesar da instituição já possuir alguns documentos de planejamento que versem sobre essa temática (PDI e PLS). Além disso, observou-se que, no geral, os técnicos administrativos possuem conhecimento limitado a respeito de instrumentos essenciais que norteiam a administração pública na implantação de práticas de sustentabilidade, fato que pode acarretar dificuldades no estabelecimento de ações nesse sentido. Sugeriram a criação de mecanismos de sensibilização e capacitação sobre o referido tema.

\section{Discussão}

Face os estudos apresentados, foi possível evidenciar que nas IES localizadas no Canadá a aprendizagem formal para a sustentabilidade está presente, considerando que foram expostos quatro modelos de indicadores para a sustentabilidade em IES. Enquanto isso, no Brasil, a A3P e a ISO 14001 (ABNT, 2015) têm sido as principais diretrizes para os processos formais se sustentabilidade em IES. Nesse sentido, foi destacada a importância dos modelos de indicadores formais para padronizar as ações de sustentabilidade nas IES. Contudo, são as práticas das IES que traduzem as suas ações reais para a sustentabilidade, resultantes da intersecção entre a aprendizagem formal e a aprendizagem informal (Flach e Antonello, 2011). 
Enfatizando a perspectiva informal da aprendizagem baseada em práticas, no Canadá o trabalho de Clarke (2006) dividiu as práticas para a sustentabilidade em três categorias: acadêmica, operacional e financeira. Mais precisamente, Clarke (2006) também conseguiu identificar as compras sustentáveis e as iniciativas de compostagem como práticas realizadas na IES. Mitchell (2011) apontou a cogeração de energia e as construções verdes como práticas vivenciadas.

No Brasil, os estudos foram mais exploratórios. Os artigos estudados procuraram descobrir e avaliar o que vem sendo feito pelas universidades, sem saber ao certo quais as práticas seriam encontradas, conforme expuseram Engelman et al. (2009), Machado et al. (2013) e Sgarbi et al. (2013). Engelman et al. (2009) apontaram algumas práticas que são decorrentes de processos formais de aprendizagem, como a realização de treinamentos ou a elaboração de documentos, percebidos na constituição de departamentos ou áreas de trabalho para a gestão ambiental. Em se tratando das práticas, os autores nacionais destacaram algumas que refletem também as dimensões abordadas nos indicadores canadenses, porém com maior detalhamento, tais como: redução no consumo de água e energia elétrica; disposição e tratamento adequado de resíduos; campanhas para a redução do uso de copos descartáveis; compostagem para resíduos orgânicos, etc.

No Canadá, os indicadores foram propostos por organizações sem fins lucrativos, apresentando dimensões que direcionam as práticas das IES. No Brasil prevalece aqui a ISO 14001 (ABNT, 2015) como norteadora das práticas e assim, por ser uma norma abrangente, da maior liberdade para que as IES definam os seus aspectos ambientais de maior relevância, predominando, portanto, a aprendizagem informal na interação com a aprendizagem formal. Condição essa que permite mais possibilidades para a emergência de novas práticas, o que não faz a realidade nacional tão diversa daquela observada no Canadá por Clarke (2006), quando a autora observou que para os Sistemas de Gestão Ambiental o modelo de estratégias emergentes é mais adequado para a realidade do que a abordagem de estratégia deliberada. Valorizando assim as relações sociais e a aprendizagem não intencional, abrindo caminhos para as comunidades de prática, quando as próprias instituições podem definir sobre a formalização dos seus processos relativos à sustentabilidade (Elkjaer, 2011).

Vale reconhecer as comunidades de práticas orientadas para a promoção da sustentabilidade nas Instituições de Ensino Superior. Essas comunidades que não podem ser impostas, mas sim estimuladas, cultivadas e valorizadas (Jacobi et al., 2011). Como percebido por Bilodeau (2014) na Universidade de British Columbia, Campus Okanagan, onde um campus conseguiu integrar a sustentabilidade às suas práticas de negócio, planos e relatórios. Nesta universidade a sustentabilidade tem sido percebida como uma direção para a inovação, corroborando com a proposição de que em uma comunidade de práticas existem possibilidades de interação e relações entre indivíduos em torno de um tópico (Antonello, 2005). No Brasil, as IES estudadas, apesar de algumas delas terem sido avaliadas por algum modelo específico de indicador, nenhuma delas os adotam como modelo formal. Apesar desta realidade, Engelman et al. (2009) e Machado et al. (2013) conseguiram elencar uma lista de práticas observadas nas IES, essas provavelmente surgiram da interação entre os membros de um dado contexto que os levou à reprodução dessas práticas, como, por exemplo, a coleta seletiva, a minimização do uso do uso de papel, água, energia e combustível, garantindo que o que está sendo praticado atualmente possa produzir efeitos sociais em longo prazo (Gherardi 2012).

Foi possível perceber que a aprendizagem relativa à sustentabilidade nas IES mediante a proposição de modelos de indicadores contribui no entendimento dos preceitos de Nicolini e Meznar (1995), quando os autores visualizaram a aprendizagem organizacional como uma construção social que transforma a cognição adquirida em conhecimento abstrato responsável. Nesse sentido, esses indicadores vêm sendo 
construídos e reavaliados para que de alguma forma possam ser empregados em diferentes realidades das estruturas de ensino.

Mediante os modelos apresentados, tanto para o Canadá, quanto para o Brasil é possível sugerir que as IES que adotam um mesmo modelo de indicadores teriam pontos comuns no processo de aprendizagem, pois a aprendizagem formal, obtida por treinamentos e padronização, tornaria as práticas mais homogêneas.

\section{Considerações finais}

Os trabalhos nacionais tiveram por propósito avaliar uma situação ambiental que já era existente nas IES, não foram observados artigos que relatassem, por exemplo, o processo de implantação de práticas sustentáveis, todos tiveram por finalidade avaliar as práticas a partir de indicadores. No Brasil não existem normatizações que regulamentem essa questão. Até mesmo a legislação é genérica, como é o caso da Política Nacional de Resíduos Sólidos (Lei no 12.305/2010) (Brasil, 2010), ou o Decreto no 5.940/2006 (Brasil, 2006), que regulamenta o correto descarte de resíduos provenientes da administração pública, bem como o recente modelo da A3P denominado Gestão Socioambiental nas IES Públicas. Nesse caso as IES privadas não teriam a mesma obrigação que as IES públicas. No Canadá, o uso de indicadores padronizados aconteceu em dois trabalhos, Beringer (2006) e Kamal e Asmuss (2013), nos demais valorizaram as práticas emergentes e a cultura empreendedora, dando suporte ao desenvolvimento sustentável.

As universidades, assim como outras empresas prestadoras de serviços não têm os seus aspectos ambientais tão facilmente observáveis, tal qual as empresas de manufatura (Tauchen e Brandli, 2006; Otero, 2010). Diante dessa realidade, um campus sustentável pode comunicar as suas ações sobre a sua responsabilidade local e global para proteger e realçar a saúde e o bem-estar dos seres humanos e ecossistemas. Abrangendo o conhecimento da comunidade universitária para destacar os desafios ecológicos e sociais do presente e para o futuro (Cole, 2003).

Em que pese os modelos de indicadores apresentados, a aprendizagem para a sustentabilidade das IES no Brasil e no Canadá apresentam similaridade e diferenças. Ambas iniciativas são recentes, mas as práticas podem variar dependendo da localização geográfica, dimensão, condições do ambiente local, capacidade de aproveitamento de oportunidades, cooperação interinstitucional e capacidade de construção de parcerias (Machado et al., 2013).

A partir destes estudos sobre a sustentabilidade ambiental em IES no Canadá e no Brasil, considera-se que as IES estão evoluindo em relação à adoção de práticas sustentáveis. Para pesquisas futuras comparar o Brasil com outras regiões do mundo também parece relevante, buscando assim as melhores práticas que possam ser adotadas pelas nossas IES, resguardando as devidas diferenças sociais e culturais.

\section{Conflito de interesses}

As autoras declaram não haver conflitos de interesse.

\section{Referências}

AASHE - Association for the Advancement of Sustainability in Higher Education. 2015. Disponível em: <http://www.aashe.org>. Acesso em: 10 fev. 2020.

ABNT - Associação Brasileira de Normas Técnicas. ABNT NBR ISO 14001:2015 Sistemas de gestão ambiental - Requisitos com orientações para uso. Rio de Janeiro: ABNT, 2015. 
Antonello, C. S. A metamorfose da aprendizagem organizacional: uma revisão crítica. In: Ruas, R. L.; Antonello, C. S.; Boff, L. H. (Orgs.). Aprendizagem organizacional e competências. Porto Alegre: Bookman, 2005. p. 12-33.

Antonello, C. S.; Godoy, A. S. A encruzilhada da aprendizagem organizacional: uma visão multiparadigmática. Revista de Administração Contemporânea, v. 14, n. 2, p. 310-332, 2010. https://doi.org/10.1590/S1415-65552010000200008

Antonello, C. S.; Godoy, A. S. Uma agenda brasileira para os estudos em aprendizagem organizacional. Revista de Administração de Empresas, v. 49, n. 3, p. 266-281, 2009. https://doi.org/10.1590/S0034-75902009000300003

Barr, P. S.; Stimpert, J. L.; Huff, A. S. Cognitive change, strategic action, and organizational renewal. Strategic Management Journal, v. 13, p. 15-36, 1992. https://doi.org/10.1002/smj.4250131004

Beringer, A. Campus sustainability audit research in Atlantic Canada: pioneering the campus sustainability assessment framework. International Journal of Sustainability in Higher Education, $\quad$ v. 7, $\quad$ n. 4, p. 437-455, 2006. https://doi.org/10.1108/ 14676370610702235

Bilodeau, L.; Podger, J.; Abd-El-Aziz, A. Advancing campus and community sustainability: Strategic alliances in action. International Journal of Sustainability in Higher Education, v. 15, n. 2, p. 157-168, 2014. https://doi.org/10.1108/IJSHE-06-2012-0051

Brasil. Decreto no 5.940, de 25 de outubro de 2006. Institui a separação dos resíduos recicláveis descartados pelos órgãos e entidades da administração pública federal direta e indireta, na fonte geradora, e a sua destinação às associações e cooperativas dos catadores de materiais recicláveis, e dá outras providências. Disponível em: <http://www.planalto.gov.br/ccivil_03/_Ato2004-2006/2006/Decreto/D5940.htm>.

Acesso em: 10 fev. 2020.

Brasil. Lei no 12.035, de 02 de agosto de 2010. Institui a Política Nacional de Resíduos Sólidos; altera a Lei no 9.605, de 12 de fevereiro de 1998; e dá outras providências. Disponível em: <http://www.planalto.gov.br/ccivil_03/_ato2007-2010/2010/lei/ 112305.htm>. Acesso em: 10 fev. 2020.

Clarke, A. The campus environmental management system cycle in practice: 15 years of environmental management, education and research at Dalhousie University. International Journal of Sustainability in Higher Education, v. 7, n. 4, p. 374-389, 2006. https://doi.org/10.1108/14676370610702181

Cohen, M. D. Individual learning and organization a routine: Emerging connections. Organization Science, v. 2, n. 1, p. 135-139, 1991. https://doi.org/10.1287/orsc.2.1.135

Cole, L. Assessing sustainability on Canadian university campuses: Development of a campus sustainability assessment framework. Victoria: Royal Roads University, 2003. (M. A. thesis).

Conlon, T. J. A review of informal learning literature, theory and implications for practice in developing global professional competence. Journal of European Industrial Training, v. 28, n. 2/4, p. 283-295, 2004. https://doi.org/10.1108/03090590410527663

Cook, S. D. N.; Yanow, D. Culture and organizational learning. Journal of Management Inquiry, v. 2, n. 4, p. 373-390, 1993. https://doi.org/10.1177/105649269324010

Cyert, R. M.; March, J. G. A behavioral theory of the firm. New Jersey: Englewook Cliffs, 1963. 
Easterby-Smith, M.; Araujo, L. Aprendizagem organizacional: oportunidades e debates atuais. In: Easterby-Smith, M.; Burgoyne, J.; Araujo, L. Aprendizagem organizacional e organização de aprendizagem: desenvolvimento na teoria e na prática. São Paulo: Atlas, 2001.

Elkington, J. Cannibals with forks: The triple bottom line of 21st century business. Oxford: Capstone Publishing, 1999.

Elkjaer, B. Social learning theory: Learning as participation in social process. In: EasterbySmith, M.; Lyles, M. (Orgs.). The Blastocele handbook of organizational learnig and knowledge management. London: Blackwell, 2003. p. 38-53.

Engelman, R.; Guisso, R. M.; Fracasso, E. M. Ações de gestão ambiental em instituições de ensino superior: o que tem sido feito. Revista de Gestão Social e Ambiental, v. 3, n. 1, p. 22-33, 2009. https://doi.org/10.24857/rgsa.v3i1.115

Flach, L.; Antonello, C. S. Organizações culturais e a aprendizagem baseada em práticas. Cadernos EBAPE.BR, v. 9, n. 1, p. 155-175, 2011. https://doi.org/10.1590/S167939512011000100010

Fonseca, A.; MacDonald, A.; Dandy, E.; Valenti, P. The state of sustainability reporting at Canadian universities. International Journal of Sustainability in Higher Education, v. 12, n. 1, p. 22-40, 2011. https://doi.org/10.1108/14676371111098285

Gherardi, S. How to conduct a practice-base study: Problems and methods. Cheltenham Glos, UK: Edward Elgar, 2012. https://doi.org/10.4337/9780857933386

Gherardi, S. Organizational learning: The sociology of practice. In: Easterby-Smith, M.; Lyles, M. Handbook of organizational learning \& knowledge management. 2 ed. New York: Wiley, 2011.

Hedberg, B. How organizations learn and unlearn. In: Nystrom, P.; Starbuck, W. (Orgs.). Handbook of organization design. Oxford: Oxford University, 1981. p. 3-27.

Huber, G. P. Organizational learning: The contributing process and the literatures. Organization Science, v. 2, n. 1, p. 88-115, 1991.

Jacobi, P. R.; Raufflet, E.; Arruda, M. P. Educação para a sustentabilidade no Cursos de Administração: reflexão sobre paradigmas e práticas. Revista de Administração

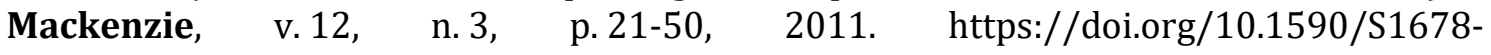
69712011000300003

Kamal, A. S.; Asmuss, M. Benchmarking tools for assessing and tracking sustainability in higher educational institutions: Identifying an effective tool for the University of Saskatchewan. International Journal of Sustainability in Higher Education, v. 14, n. 4, p. 449-465, 2011. https://doi.org/10.1108/IJSHE-08-2011-0052

Kruger, S. D.; Freitas, C. L.; Pfitscher, E. D.; Petri, S. M. Gestão ambiental em instituição de ensino superior: uma análise da aderência de uma instituição de ensino superior comunitária aos objetivos da agenda ambiental na administração pública (A3P). Revista GUAL, v. 4, n. 3, p.44-62, 2011. https://doi.org/10.5007/1983-4535.2011v4n3p44

Lave, J.; Wenger, E. Situated learning: Legitimate peripheral participation. New York: Cambridge University Press, 1991.

Lyles, M. A.; Easterby-Smith, M. Organizational learning and knowledge management: Agendas for future research. In: Easterby-Smith, M.; Lyles, M. (Orgs). The Blackwell handbook of organizational learning and knowedge management. London: Blackwell, 2003. p. 639-652. 
Machado, R. E.; Fracasso, E. M.; Tometich, P.; Nascimento, L. F. Práticas de gestão ambiental em universidades brasileiras. Revista de Gestão Social e Ambiental, v. 7, n. 3, p. 37-51, 2013. https://doi.org/10.24857/rgsa.v7i3.740

March, J. G. Exploration and exploitation in organization learning. Organization Science, v. 2, n. 1, p. 71-87, 1991.

Marsick, V. J.; Watkins, K. E. Informal and incidental learning. New Directions for Adult and Continuing Education, v. 89, p. 25-34, 2001. https://doi.org/10.1002/ace.5

Mitchell, R. M. Sustaining change on a Canadian campus: Preparing Brock University for a sustainability audit. International Journal of Sustainability in Higher Education, v. 12, n. 1, p. 7-21, 2011. https://doi.org/10.1108/14676371111098276

Munro, A.; Marcus, J.; Dolling, K.; Robinson, J.; Wahl, J. Combining forces: Fostering sustainability collaboration between the City of Vancouver and the University of British Columbia. International Journal of Sustainability in Higher Education, v. 17, n. 6, p. 812-826, 2016. https://doi.org/10.1108/IJSHE-04-2015-0082

Nicolini, D. Practice theory, work \& organization: An introduction. Oxford: Oxford University, 2013.

Nicolini, D.; Meznar, M. B. The social construction of organizational learning: Conceptual and practical issues in the fied. Human Relations, v. 48, n. 7, p.725-746, 1995. https://doi.org/10.1177/001872679504800701

OIUDSMA - Associação Internacional de Universidades para o Desenvolvimento Sustentável e o Meio Ambiente. 2015. Disponível em: <http://www.oiudsmanimad.ufpr.br>. Acesso em: 10 fev. 2020.

Otero, G. G. P. Gestão ambiental em instituições de ensino superior: práticas dos campi da Universidade de São Paulo. São Paulo: Programa de Pós-Graduação em Ciência Ambiental, Universidade de São Paulo, 2010. (Dissertação de mestrado).

Patriotta, G. Organizational knowledge in the making: How firms create, use, and institutionalize knowledge. Oxford: Oxford University Press, 2003.

Peixoto, C. S. B. S.; Moraes Filho, R. A.; Moraes, I. C.; Vieira, L. G. H. Práticas sustentáveis: estudo de caso em uma instituição de ensino superior. Revista GUAL, v. 12, n. 2, p. 230 252, 2019. https://doi.org/10.5007/1983-4535.2019v12n2p30

Reatto, D.; Godoy, A. S. A produção sobre aprendizagem informal nas organizações no Brasil: mapeando o terreno e rastreando possibilidades futuras. Revista Eletrônica de Administração, v. 80. n. 1, p. 57-88, 2015. https://doi.org/10.1590/1413-2311.0102014. 47369

Richardson, R. J. Pesquisa social: métodos e técnicas. 3. ed. São Paulo: Atlas, 1999.

Rocha, S. K.; Pfitscher, E. D.; Carvalho, F. N. Sustentabilidade ambiental: estudo em uma instituição de ensino superior pública catarinense. Revista de Gestão Ambiental e Sustentabilidade, v. 4, n. 1, p. 46-58, 2015. https://doi.org/10.5585/geas.v4i1.162

Sachs, I. Estratégias de transição para o século XXI. In: Bursztyn, M. (Org.). Para pensar o desenvolvimento sustentável. São Paulo: Brasiliense, 1993.

Sgarbi, M.; Schlosser, R. T.; Campani, D. B. Implantação do Sistema de Gestão Ambiental em uma universidade pública no Rio Grande do Sul, Brasil. AUGMDOMUS, v. 5, p. 120-140, 2013. 
SYC - Sierra Youth Coalition. 2015. Disponível em: <http://www.syc-cjs.org>. Acesso em: 10 fev. 2020.

Takahashi, A. R. W. Descortinando os processos da aprendizagem organizacional no desenvolvimento de competências em instituições de ensino. São Paulo: Faculdade de Economia, Administração e Contabilidade, Universidade de São Paulo, 2007. (Tese de doutorado).

Tauchen, J.; Brandli, L. L. A gestão ambiental em instituições de ensino superior: modelo para implantação em campus universitário. Gestão \& Produção, v. 13, n. 3, p. 503-515, 2006. https://doi.org/10.1590/S0104-530X2006000300012

Termignoni, L. D. Framework de sustentabilidade para instituições de ensino superior comunitárias. Porto Alegre: Pontifícia Universidade Católica do Rio Grande do Sul, 2012. (Dissertação de mestrado em Administração).

The Halifax Declaration. 1991. Disponível em: <http://www.iisd.org/educate/declarat/ halifax.htm>. Acesso em: 10 fev. 2020.

The Swansea Declaration. 1993. Disponível em: <http://www.iisd.org/educate/declarat/ swansea.htm>. Acesso em: 10 fev. 2020.

The Talloires Declaration. 1990. Disponível em: <http://ulsf.org/programs_ talloires.html>. Acesso em: 10 fev. 2020.

Tsang, E. W. K. Organizational learning and the learning organization: a dichotomy between descriptive and prescriptive research. Human Relations, v. 50, n. 1, p. 73-89, 1997. https://doi.org/10.1177/001872679705000104

Watkins, K. E.; Marsick, V. J. Towards a theory of informal and incidental learning in organizations. International Journal of Lifelong Education, v. 11, n. 4, p. 287-300, 1992. https://doi.org/10.1080/0260137920110403

Informação da Licença: Este é um artigo Open Access distribuído sob os termos da Licença Creative Commons Attribution, que permite uso irrestrito, distribuição e reprodução em qualquer meio, desde que a obra original seja devidamente citada. 